
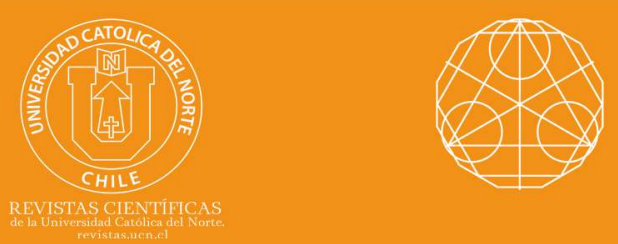

\title{
The essential norm of multiplication operators acting on Orlicz sequence spaces
}

Julio C. Ramos-Fernández ${ }^{1}$ (1) orcid.org/0000-0002-7329-5263

Margot Salas-Brown ${ }^{2}$ (1) orcid.org/0000-0001-7202-8916

${ }^{1}$ Universidad Distrital Francisco José de Caldas, Facultad Tecnológica, Bogotá, Colombia. - jcramosf@udistrital.edu.co

${ }^{2}$ Universidad del Rosario, Escuela de Ingeniería, Ciencia y Tecnología, Matemáticas Aplicadas y Ciencias de la Computación, Bogotá, Colombia.

margot.salas@urosario.edu.co

Received: July 2019 | Accepted: September 2020

\section{Abstract:}

We calculate the measure of non-compactness or the essential norm of the multiplication operator Mu acting on Orlicz sequence spaces $l^{\varphi}$. As a consequence of our result, we obtain a known criteria for the compactness of multiplication operator acting on $l^{\varphi}$.

Keywords: Multiplication operator; Orlicz sequence spaces; Banach lattice; Essential norm.

MSC (2020): 47B38, 46E30, 46B42.

\section{Cite this article as (IEEE citation style):}

J. C. Ramos-Fernández and M. Salas-Brown, "The essential norm of multiplication operators acting on Orlicz sequence spaces", Proyecciones (Antofagasta, On line), vol. 39, no. 6, pp. 1407-1414, Dec. 2020, doi: 10.22199/issn.0717-6279-2020-06-0086.

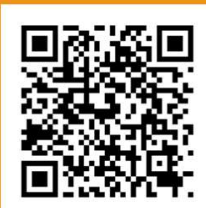

Article copyright: (C) 2020 Julio C. Ramos-Fernández, and Margot Salas-Brown. This is an open access article distributed under the terms of the Creative Commons License, which permits unrestricted use and distribution provided the original author and source are credited. 


\section{Introduction}

Let $(\Omega, \Sigma)=(\Omega, \Sigma, \mu)$ be a complete $\sigma$-finite measure space and let $L^{0}(\mu)$ denote the space of all $\mu$-equivalence classes of $\Sigma$-measurable functions on $\Omega$ with the topology of convergence in measure on $\mu$-finite sets. A Banach space $\left(X,\|\cdot\|_{X}\right)$ consisting of equivalence classes modulo equality almost everywhere of locally integrable real-valued functions on $\Omega$ is said to be a Köthe space on $(\Omega, \Sigma)$ if the following conditions hold:

(i) If $|f(t)| \leq|g(t)|$ a.e. on $\Omega$, with $f$ measurable and $g \in X$, then $f \in X$ and $\|f\|_{X} \leq\|g\|_{X}$ (often this property is refer as the space is solid).

(ii) For every $A \in \Sigma$ with $\mu(A)<\infty$, the characteristic function $\mathbf{1}_{A}$ of $A$ belongs to $X$.

A function $f$ is locally integrable if it belongs to $L^{0}(\mu)$ and $\int_{A}|f| d \mu<\infty$ for each $A \in \Sigma$ such that $\mu(A)<\infty$. Every Köthe space is a Banach lattice in the obvious order ( $g \geq 0$ if $g(t) \geq 0$ a.e.). By (i) every Köthe space is $\sigma$ order complete (see [8]). If in addition the unit ball $B_{X}=\left\{f:\|f\|_{X} \leq 1\right\}$ is closed in $L^{0}(\mu)$, then we say that $X$ has the Fatou property. A Köthe space defined on $\left(N, 2^{N}, \mu\right)$ with the counting measure $\mu$ is called a Köthe sequence space (on $N)$.

The Köthe dual $X^{\prime}$ of the Köthe space $X$ defined on $(\Omega, \Sigma)$ ia a Banach space which can be identified with the space of all functional possessing an integral representation, that is,

$$
X^{\prime}=\left\{g \in L^{0}(\mu):\|g\|_{X^{\prime}}=\sup _{\|f\|_{X} \leq 1} \int_{\Omega}|f g| d \mu<+\infty\right\} .
$$

Ii is known that if $X$ has order continuous norm (i.e. $\left\|f_{n}\right\|_{X} \rightarrow 0$ for all sequence $\left\{f_{n}\right\} \subset X$ such that $\left.f_{n} \downarrow 0\right)$, then the dual space $X^{*}$ can be naturally identified with $X^{\prime}$ (see $[6]$ ).

Each function $u \in L^{0}(\mu)$ define un lineal operator $M_{u}$ given by $M_{u}(f)=$ $u \cdot f$, the pointwise multiplication of $u$ times $f$, which is known as multiplication operator with symbol $u$. The multiplication operator acting on Banach function spaces is a well-studied transformation. We must mention here the pioneering work Singh/Kumar in [12] and [13] on properties of multiplication operators acting on spaces of measurable functions. These authors studied the compactness and closedness of the range of multiplication operators on $L_{2}(\mu)$. A big numerous of mathematicians have extended 
or generalized the results of Singh and Kumar to important examples of Köthe spaces (we omit cites), however, the more general study of the properties of the operator $M_{u}: X \rightarrow X$ (such as the compactness and closedness of the range among others), with $X$ being a Köthe space is due to Hudzik et al. [5] (see also Castillo et al. [3]).

Related to the problem of the characterization of the compactness of multiplication operators we have the problem of to estimate its essential norm or the non-compactness measure of multiplication operators. This subject has been widely studied in the context of analytic functions. In the case of multiplication operators $M_{u}$, acting on non-atomic Köthe space, the essential norm was calculate by Castillo et al. [3] and it is an open problem calculate the essential norm of this operator in a more general sense. The essential norm of multiplication operators acting on Lorentz sequence spaces (which is a Köthe sequence space) was calculate by Castillo et al. [4]. The main objective of this article is to give a light about the value of the essential norm of multiplication operators acting on purely atomic Köthe space. More precisely, we are going to show that the result obtained by Castillo et al. in [4] is also valid for Orlicz sequence spaces, the proof of this aim is given in Section 3. The article is completed with the Section 2 where we gather some properties of the Orlicz sequence spaces.

\section{Some remarks about Orlicz sequence spaces}

We recall that a function $\varphi:[0, \infty) \rightarrow[0, \infty)$ is said to be an Orlicz function if it is a non-decreasing convex function which assume the vaue zero only at zero and such that $\lim _{t \rightarrow+\infty} \varphi(t)=+\infty$. Given an Orlicz function $\varphi$, the Orlicz sequence space $l^{\varphi}$ is the set of all real sequences $x=\{x(n)\}_{n=1}^{\infty}$ such that

$$
\sum_{n=1}^{\infty} \varphi(\lambda|x(n)|)<\infty
$$

for some $\lambda>0 . l^{\varphi}$ is a Banach space with the Luxemburg norm defined by

$$
\|x\|_{\varphi}=\inf \left\{\lambda>0: \sum_{n=1}^{\infty} \varphi\left(\frac{|x(n)|}{\lambda}\right) \leq 1\right\} .
$$

In particular, each sequence $x=\{x(n)\}_{n=1}^{\infty} \in l^{\varphi}$ belongs to $c_{0}$, the space of all real sequence converging to zero. Clearly if $x, y$ are real sequences such that $y \in l^{\varphi}$ and $|x(n)| \leq|y(n)|$ for all $n \in N$, then $x \in l^{\varphi}$ and $\|x\|_{\varphi} \leq\|y\|_{\varphi}$, 
this fact tell us that $l^{\varphi}$ is a solid space. Furthermore, if $A=\left\{n_{1}, n_{2}, \cdots, n_{m}\right\}$ is a finite subset of $N$, then the characteristic sequence $\mathbf{1}_{A}$ defined by

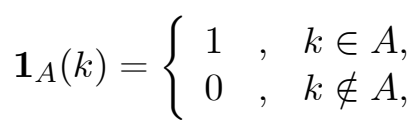

belongs to $l^{\varphi}$. Hence, $l^{\varphi}$ is a Köthe sequence space. Observe that for the set $A$ defined above, $\left\|\mathbf{1}_{A}\right\|_{\varphi}=\left(\varphi^{-1}\left(\frac{1}{m}\right)\right)^{-1}$, where $\varphi^{-1}(t)=\inf \{s>0: \varphi(s)>t\}$ is the right continuous inverse of $\varphi$.

Each Orlicz function $\varphi$ allow us to define other Orlicz function $\psi:[0, \infty) \rightarrow$ $[0, \infty)$ which is known as the complementary function to $\varphi$ and it is defined by

$$
\psi(t)=\sup \{\tau|t|-\varphi(\tau): \tau \geq 0\} .
$$

Then each $y=\{y(n)\}_{n=1}^{\infty} \in l^{\psi}$ defines a bounded linear functional $F_{y}$ on $l^{\varphi}$ by

$$
F_{y}(x)=\sum_{n=1}^{\infty} x(n) y(n), \quad x=\{x(n)\}_{n=1}^{\infty} \in l^{\varphi} .
$$

Moreover, the mapping $y \rightarrow F_{y}$ is an isometry from $l^{\psi}$ onto $\left(l^{\varphi}\right)^{*}$, and the norm dual of $l^{\varphi}$ can be identified with $l^{\psi}$. Hence the dual of the Orlicz sequence space coincides with its Köthe dual. We refer this fact as the Ries's representation theorem for the Orlicz sequence space.

We conclude this section with the following elementary properties of the canonical basis $\left\{e_{k}\right\}_{k=1}^{\infty}$ which it will be of great utility in the proof of our result. Here, for $k \in N$ fixed, $e_{k}$ is the sequence defined by

$$
e_{k}(n)=\mathbf{1}_{\{k\}}(n)= \begin{cases}1, & n=k \\ 0, & n=k .\end{cases}
$$

Then $\left\|e_{k}\right\|_{\varphi}=\left(\varphi^{-1}(1)\right)^{-1}$ for all $k \in N$ and $\left\{e_{k}\right\}_{k=1}^{\infty}$ converges weakly to zero since each $y \in l^{\psi}$ is an element of $c_{0}$. We refer the excellent book of Rao and Ren [11] for more properties of the Orlicz spaces.

\section{The main result}

Given a Banach space $\left(X,\|\cdot\|_{X}\right)$, the essential norm of a continuous linear operator $T: X \rightarrow X$, denoted by $\|T\|_{e}$, is its distance to the class $\mathcal{K}(X)$ of all compact operators on $X$, that is,

$$
\|T\|_{e}=\inf \{\|T-K\|: K \in \mathcal{K}(X)\}
$$


where $\|T\|$ denotes the operator norm of $T$, which is defined by

$$
\|T\|=\sup \left\{\|T f\|_{X}:\|f\|_{X}=1\right\} .
$$

Since $\mathcal{K}(X)$ is a closed subspace of $\mathcal{B}(X)$, the class of all bounded linear operator (with the topology induced by the operator norm), we can see that an operator $T \in \mathcal{B}(X)$ is compact if and only if $\|T\|_{e}=0$. Furthermore, $\|T\|_{e}$ is the norm of the class of $T$ in the Calkin algebra $B(X) / \mathcal{K}(X)$.

The essential norm of multiplication operators acting on Banach function spaces has not been calculated yet in the more general setting. However there are some works about this subject. If $X$ is a non-atomic order continuous Köthe space, Castillo et al. [3] proved that the essential norm of $M_{u}: X \rightarrow X$ is given by $\left\|M_{u}\right\|_{e}=\|u\|_{\infty}$, the essential supremum of $|u|$. Hence, the only compact multiplication operator acting on this kind spaces is the null operator. Motived by the idea in to find non-null compact multiplication operators, recently, have appeared interesting works about properties of multiplication operators acting on Banach sequences spaces. Arora et al. in [1] proved that a multiplication operator $M_{u}$ acting on Lorentz sequence spaces is compact if and only if $u \in c_{0}$; that is, if and only if $u(n) \rightarrow 0$ as $n \rightarrow \infty$. This last condition also characterize the compactness of this operator $M_{u}$, acting on other Köthe sequence spaces such as Orlicz-Lorentz sequence spaces [2], Cesàro sequence spaces [7], CesàroOrlicz sequence spaces [9], among others. However, the characterization of compactness (and other properties) of multiplication operators acting on any Köthe sequence spaces is due to Ramos-Fernández and Salas-Brown [10] (see also [5] and [3]). The compactness result obtained by RamosFernández and Salas-Brown in [10] can be stated as follows:

Theorem 3.1 ([10]). Let $X$ be a Köthe sequence space and suppose that $u \in l^{\infty}$. The operator $M_{u}: X \rightarrow X$ is compact if and only if $u \in c_{0}$.

Here, the product of two sequences $a=\{a(n)\}_{n=1}^{\infty}$ and $b=\{b(n)\}_{n=1}^{\infty}$ is defined by $a \cdot b=\{a(n) \cdot b(n)\}_{n=1}^{\infty}$ and the condition $u \in l^{\infty}$ characterize the continuity of the operator $M_{u}: X \rightarrow X$, where $l^{\infty}$ denotes the space of all bounded real sequence. The objective is to find some estimation of $\left\|M_{u}\right\|_{e}$ which implies Theorem 3.1. In the case of Lorentz sequence spaces $l_{(p, q)}$, Castillo et al. in [4] proved that the essential norm of the operator $M_{u}: l_{(p, q)} \rightarrow l_{(p, q)}$ is given by $\left\|M_{u}\right\|_{e}=\lim \sup _{n \rightarrow \infty}|u(n)|$ which implies the result of Arora et al. in [1]. We are going to prove that the same result is also valid for the Orlicz sequence spaces. The result we have obtained is the following: 
Theorem 3.2. Let $\varphi$ an Orlicz function and suppose that $u \in l^{\infty}$. For the multiplication operator $M_{u}: l^{\varphi} \rightarrow l^{\varphi}$ we have

$$
\left\|M_{u}\right\|_{e}=\limsup _{n \rightarrow \infty}|u(n)| .
$$

Proof. Suppose that the sequence $u=\{u(n)\}_{n=1}^{\infty}$ is bounded. For each $N \in \mathbf{N}$, we consider the set $A_{N}=\{1,2, \cdots, N\}$ and we set the sequence $u_{N}=u \cdot \mathbf{1}_{A_{n}}$, then by Theorem 3.1, the multiplication operator $M_{u_{N}}: l^{\varphi} \rightarrow$ $l^{\varphi}$ is compact. Hence

$$
\left\|M_{u}\right\|_{e} \leq\left\|M_{u}-M_{u_{N}}\right\|=\left\|M_{u-u_{N}}\right\| .
$$

But if $a=\{a(n)\} \in l^{\varphi}$ is such that $\|a\|_{\varphi}=1$, then clearly

$$
\left|\left(u(n)-u_{N}(n)\right) \cdot a(n)\right| \leq S_{N}|a(n)|
$$

for all $n \in \mathbf{N}$, where $S_{N}=\sup \{|u(k)|: k \geq N\}<\infty$. Thus, since the space $l^{\varphi}$ is solid, we conclude that the sequence $\left(u-u_{N}\right) \cdot a$ belongs to $l^{\varphi}$ and

$$
\begin{aligned}
\left\|M_{u}(a)-M_{u_{N}}(a)\right\|_{\varphi} & =\left\|\left(u-u_{N}\right) a\right\|_{\varphi} \\
& \leq S_{N}\|a\|_{\varphi}=S_{N} .
\end{aligned}
$$

Therefore $\left\|M_{u}\right\|_{e} \leq\left\|M_{u}-M_{u_{N}}\right\| \leq S_{N}$ for all $N \in \mathbf{N}$ and hence

$$
\left\|M_{u}\right\|_{e} \leq \limsup _{n \rightarrow \infty}|u(n)| .
$$

On the other hand, if $K: l^{\varphi} \rightarrow l^{\varphi}$ is any compact operator, then, since the sequence $\left\{e_{k}\right\}_{k=1}^{\infty}$ is a bounded in $l^{\varphi}$ and converges weakly to zero, we have that

$$
\lim _{k \rightarrow \infty}\left\|K\left(e_{k}\right)\right\|_{\varphi}=0 .
$$

Indeed, if (3.1) is false, then we can find a $\delta>0$ and a subsequence $\left\{e_{k_{m}}\right\}_{m=1}^{\infty}$ of $\left\{e_{k}\right\}_{k=1}^{\infty}$ such that

$$
\left\|K\left(e_{k_{m}}\right)\right\|_{\varphi} \geq \delta
$$

for all $m \in N$. Since $K: l^{\varphi} \rightarrow l^{\varphi}$ is a compact operator, then by passing to a subsequence if is necessary, we can suppose that $\left\{K\left(e_{k_{m}}\right)\right\}_{m=1}^{\infty}$ converges in $l^{\varphi}$. That is, there exists a $b \in l^{\varphi}$ such that

$$
\lim _{m \rightarrow \infty}\left\|K\left(e_{k_{m}}\right)-b\right\|_{\varphi}=0 .
$$

We are going to prove that $b=0$ which it will be a contradiction to (3.2). By Hahnn-Banach's theorem, it is enough to show that $h(b)=0$ 
for all bounded linear functionals $h$ on $l^{\varphi}$. Let $h$ be any bounded linear functional on $l^{\varphi}$, then

$$
\begin{aligned}
|h(b)| & \leq\left|h(b)-h K\left(e_{k_{m}}\right)\right|+\left|h K\left(e_{k_{m}}\right)\right| \\
& \leq\|h\|\left\|b-K\left(e_{k_{m}}\right)\right\|_{\varphi}+\left|h K\left(e_{k_{m}}\right)\right| \rightarrow 0
\end{aligned}
$$

as $m \rightarrow \infty$ since $\left\{e_{k}\right\}_{k=1}^{\infty}$ converges weakly to zero. Hence $h(b)=0$ and we have proved the claim. Next we can conclude the proof of our result. For each $k \in \mathbf{N}$, we can write

$$
\begin{aligned}
\left\|M_{u}-K\right\| & \geq\left\|M_{u}\left(\frac{e_{k}}{\left\|e_{k}\right\|_{\varphi}}\right)-K\left(\frac{e_{k}}{\left\|e_{k}\right\|_{\varphi}}\right)\right\|_{\varphi} \\
& \geq \frac{1}{\left\|e_{k}\right\|_{\varphi}}\left\|M_{u}\left(e_{k}\right)\right\|_{\varphi}-\varphi^{-1}(1)\left\|K\left(e_{k}\right)\right\|_{\varphi} \\
& =|u(k)|-\varphi^{-1}(1)\left\|K\left(e_{k}\right)\right\|_{\varphi} .
\end{aligned}
$$

Thus taking the limit when $k \rightarrow \infty$ and using (3.1), we conclude that

$$
|| M_{u}-K \| \geq \limsup _{k \rightarrow \infty}|u(k)|
$$

and therefore $\left\|M_{u}\right\|_{e} \geq \limsup _{n \rightarrow \infty}|u(n)|$ since the compact operator $K$ on $l^{\varphi}$ was arbitrary. This finishes the proof.

As an important consequence we have the following result (we don't found any reference in the web for this fact):

Corollary 3.3. Let $\varphi$ an Orlicz function and suppose that $u \in l^{\infty}$. The multiplication operator $M_{u}: l^{\varphi} \rightarrow l^{\varphi}$ is compact if and only if $u \in c_{0}$.

We finish this note observing that the proof of our main result only depend on the properties of the Luxemburg norm and on the properties of the canonical basis which implies that our result is also valid in a more general context.

\section{References}

[1] S. C. Arora, G. Datt, and S. Verma, "Operators on Lorentz sequence spaces", Mathematica bohemica, vol. 134, no. 1, pp. 87-98, 2009. [On line]. Available: https:/ / bit.ly/ 31H7Chq

[2] P. Bala, A. Gupta and N. Bhatia, "Multiplication operators on OrliczLorentz sequence spaces", International journal of mathematical analysis, vol. 7, no. 29-32, pp. 1461-1469, 2013, doi: 10.12988/ ijma.2013.3360 
[3] R. E. Castillo, H. Rafeiro, J. C. Ramos-Fernández, and M. Salas-Brown, "Multiplication operator on Köthe spaces: measure of non-compactness and closed range", Bulletin Malaysian Mathematics Sciences Society, vol. 42, no. 4, pp. 1523-1534, Jul. 2019, doi: $10.1007 /$ s40840-017-0562-0

[4] R. E. Castillo, J. C. Ramos-Fernández, and M. Salas-Brown, "The essential norm of multiplication operators on Lorentz sequence spaces", Real analysis exchange, vol. 41, no. 1, pp. 245-251, 2016. [On line]. Available: https:/ / bit.ly/ 35yrV1L

[5] H. Hudzik, R. Kumar, and R. Kumar, "Matrix multiplication operators on Banach function spaces", Proceedings of the Indian Academy of Sciences - Mathematical Science, vol. 116, no. 1, pp. 71-81, Feb. 2006, doi: $10.1007 /$ BF02829740

[6] L. V. Kantorovich and G. P. Akilov, Functional analysis, 2nd ed. Moscow: Nauka, 1977.

[7] B. S. Komal, S. Pandoh, and K. Raj, "Multiplication operators on Cesàro sequence spaces", Demonstratio mathematica, vol. 49, no. 4, pp. 430-436, Nov. 2016, doi: 10.1515/ dema-2016-0037

[8] P. Lin, Köthe-Bochner function spaces, Boston, MA: Birkhäuser, 2004.

[9] K. Raj, C. Sharma, and S. Pandoh, "Multiplication operators on CesàroOrlicz sequence spaces", Fasciculi mathematici, vol. 57, no. 1, pp. 137-145, 2016, doi: 10.1515/ fascmath-2016-0021

[10] J. C. Ramos-Fernández and M. Salas-Brown, "On multiplication operators acting on Köthe sequence spaces", Afrika matematika, vol. 28, no. 3-4, pp. 661-667, Jun. 2017, doi: 10.1007/ s13370-016-0475-3

[11] M. M. Rao and Z. D. Ren, The theory of Orlicz spaces, New York, NY: Marcel Dekker, 1991.

[12] R. K. Singh and A. Kumar, "Multiplication operators and composition operators with closed ranges", Bulletin Australian Mathematical Society, vol. 16, no. 2, pp. 247-252, Apr. 1977, doi: $10.1017 /$ S0004972700023261

[13] R. K. Singh and A. Kumar, "Compact composition operators", Journal of the Australian Mathematical Society. Series A. Pure Mathematics, vol. 28, no. 3, pp. 309-314, Nov. 1979, doi:10.1017/ S1446788700012258 